\title{
Inhalation of Immuno-Therapeutics/- Prophylactics to Fight Respiratory Tract Infections: An Appropriate Drug at the Right Place!
}

\author{
Thomas Sécher ${ }^{1,2 \dagger}$, Alexie Mayor ${ }^{1,2 \dagger}$ and Nathalie Heuzé-Vourc' $h^{1,2 *}$ \\ ${ }^{1}$ INSERM U1100, Centre d'Etude des Pathologies Respiratoires, Tours, France, ${ }^{2}$ Centre d'Etude des Pathologies \\ Respiratoires, Université de Tours, Tours, France
}

Keywords: respiratory infection, biopharmaceutics, immune-pharmaceutics, topical delivery, inhalation

\section{INTRODUCTION}

Respiratory tract infections (RTIs) are the third leading cause of morbidity and mortality worldwide, accounting for $\sim 4.25$ million deaths in 2010, in either children, adults or the elderlies. RTIs encompass acute infections of the upper (rhinosinusitis, ...) and lower airways (pneumonia, bronchiolitis, ...) and are also inherently associated with chronic diseases such as chronic obstructive pulmonary disease (COPD) and cystic fibrosis (CF). In addition to premature mortality, RTIs result in a huge burden on the society considering quality-adjusted life year loss and additional pressure on the overwhelmed healthcare systems, thereby representing a major public health issue.

Antimicrobial chemotherapies (e.g., antibiotics, antivirals) are the standard interventions to

Giuseppe Andrea Sautto, University of Georgia, United States

Reviewed by:

Yuan Tian,

La Jolla Institute for Immunology (LJI), United States

*Correspondence: Nathalie Heuzé-Vourc'h nathalie.vourch@med.univ-tours.fr

tThese authors have contributed equally to this work

Specialty section:

This article was submitted to Vaccines and Molecular Therapeutics,

a section of the journal

Frontiers in Immunology

Received: 12 September 2019 Accepted: 12 November 2019 Published: 29 November 2019

Citation:

Sécher T, Mayor A and Heuzé-Vourc'h N (2019) Inhalation of Immuno-Therapeutics/-Prophylactics to Fight Respiratory Tract Infections: An Appropriate Drug at the Right Place! Front. Immunol. 10:2760. doi: 10.3389/fimmu.2019.02760 prevent and to treat respiratory infections. However, their effectiveness is declining due to increased pathogen resistance, urging alternative or complementary strategies to reinforce the anti-infectious arsenal to fight RTIs. Among those under evaluation, immunomodulatory agents (immunopharmaceutics) like therapeutic antibodies $(\mathrm{Ab})$ or other therapeutic proteins and vaccines may offer novel opportunities for the prevention and treatment of RTIs, by targeting pathogens and boosting the host immune system. When used in a preventive way in patients at risk, or therapeutically to stop or to limit the spread of infection, both immunopropylactics and immunotherapeutics are administered through parenteral routes (including intravenous, subcutaneous, and intramuscular) (Table 1). As demonstrated in preclinical studies, parenteral delivery may not be optimal for large molecular weight entities to treat respiratory diseases $(1,2)$ since they poorly reach the lung compartment. In contrast, inhalation, comprising the intranasal and oral respiratory routes, targets drugs into the respiratory tract. Currently, inhalation is used both for locally- and systemically-acting drugs as it allows a straight delivery to the diseased organ and a portal to the blood circulation, considering the extensive alveolus-capillary interface. By providing a better therapeutic index, inhalation is the gold standard for small molecules, delivered topically as an aerosol, like corticosteroids/steroids, decongestants or bronchodilators for the treatment of asthma, rhinosinusitis or COPD. Besides, it is also indicated for antibiotics (nasal and oral inhalation), a local-acting protein therapeutic-Dornase alpha (Pulmozyme ${ }^{\circledR}$, oral inhalation), a mucolytic agent for patients with CF and an influenza live vaccine (FluMist ${ }^{\circledR}$ Quadrivalent, nasal inhalation).

\section{LOCAL-ACTING IMMUNOPHARMACEUTICS DELIVERED BY INHALATION}

There are accumulating evidences that administration of anti-infectious Abs, protein therapeutics (e.g., cytokines) and vaccines, to the upper and/or lower respiratory tract by inhalation, 
with the purpose of inducing a local action, is effective (3). Several preclinical studies showed the superiority of immunopharmaceutics administered topically to the respiratory tract in RTI models, in both therapeutic and prophylactic regimens. For instance, inhalation of antiinfectious Abs in models of pneumonia using Pseudomonas aeruginosa or influenza virus conferred higher protection and greater therapeutic response, respectively, compared to parenteral route administration $(4,5)$. Besides, other immunoprophylactics delivered through the respiratory route such as immunocytokines (e.g., IL-7 Fc) (6) and live-attenuated vaccines (7) showed superior performances over conventional routes against airborne viruses, in mice and non-human primates, respectively. Conversely, restricting the response to the site of action for pleiotropic molecules (e.g., IL-7 Fc), envisioned as adjuvant molecule, may reduce systemic side-effects. As reported for anti-infectious Abs, the inhaled route may also enable a higher efficacy with a lower dose (4). This means that the inhaled route may allow, in the future, to alleviate the financial burden of immunopharmaceutics (in particular Abs), which may exceed the ability of both individual patients and the healthcare systems to sustain them. Additional benefit of the inhaled route includes its non-invasiveness, offering a better comfort for patients, in particular those with chronic respiratory infections, and thus preventing additional healthcare costs. Besides, needle-free vaccination may prevent the risk of cross-contamination and facilitate mass vaccination efforts.

However, beyond clear preclinical proofs of concept and obvious theoretical advantages of the inhalation route for immunotherapeutics and -prophylactics, few of these benefits have materialized in the clinic (Table 1). Except for Flumist ${ }^{\circledR}$ Quadrivalent (Astrazeneca), an intranasal live attenuated influenza vaccine, other marketed immunoprophylactics vaccines (including those against Streptococcus pneumoniae, Haemophilus influenza, Mycobacterium tuberculosis, Bordetella pertussis or measles and Ab (anti-RSV Pavilizumab)-are administered systemically. Similarly, none of the protein therapeutics is given by inhalation. Recently, Ablynx developed an inhaled anti-RSV trimeric nanobody ${ }^{\circledR}$ (ALX-0171) for therapeutic purposes. Despite promising results in several animal models, the development has been interrupted due to insufficient evidences of efficacy during Phase 2 trial in children (in Japan). In 2019, only one phase 2 trial with an inhaled antiinfectious protein therapeutics is still ongoing (NCT03570359) assessing the efficacy of topical lung delivery of IFN- $\beta 1$ a (SNG001, Synairgen/Astrazeneca), as an immunostimulant to treat COPD exacerbations. Overall, this highlights the complexity of developing inhaled biopharmaceuticals and points out the persisting hurdles (Figure 1).

\section{CHALLENGES FOR THE DEVELOPMENT OF INHALED IMMUNE- THERAPEUTICS/PROPHYLACTICS}

The instability of immunopharmaceutics and vaccines often emerges as a challenge for inhalation delivery. Therapeutic proteins and vaccines are sensitive to various conditions which may alter their structure, thereby decrease their activity. Delivering a drug through the inhalation route implies either spraying, drying or aerosolizing, which is associated with multiple stresses (shearing, temperature, air/liquid interface, ...) potentially deleterious as widely discussed elsewhere $(8,9)$. To deal with this, both the device used for the generation of the aerosol and the formulation must be adapted, as successfully reported for Ab-based therapeutics $(3,10)$. However, the excipients must be adapted for respiratory delivery. The choice of mucosal-licensed adjuvants, which should be exempt of intrinsic immune-toxicity, and the instability of the associated carrier [e.g., nanoparticles, liposomes, immune stimulating complexes (ISCOMs)] is particularly challenging for the inhalation delivery of vaccines, especially those of the latest generation (e.g., T, B-epitope-based vaccines). The drug and device combination yields proper aerodynamical properties (particle size, flow rate, ...) to achieve the anticipated deposition in the appropriate area of the respiratory tract. Indeed, appropriate deposition to the anatomical site is mandatory to ensure an optimal efficacy. On one hand, this depends on the drug formulation (e.g., surface tension and viscosity for liquid formulation) (11) and device performances to allow the therapeutic agent to reach the site of infection (Figure 1), by this means the microbe. For lung infections, most pneumonia consists of an aggregate of trachea-bronchitis and alveolar infections. Theoretically, this clinical condition may benefit from a uniform distribution all over the lungs, with a polydisperse aerosol (ranging $1-5 \mu \mathrm{m}$ ). However, several pathogens are associated with specific anatomic localization, like S. pneumoniae, which is mainly found in the alveolar spaces, thereby requiring low-size aerosols $(<2-3 \mu \mathrm{m})$ to be targeted. On the other hand, delivery to the mucosalassociated lymphoid tissue (MALT), located in the tonsils, would be more adapted for vaccines to induce an adaptive immune response, since MALT plays a central role in the primary respiratory immune defense (Figure 1).

Biological barriers are additional hurdles to overcome and apply to all inhaled anti-infectious agents (12). First, a pathogen can "hide" itself inside host cells like M. tuberculosis in alveolar macrophages, thus being more difficult to be targeted by immunopharmaceutics. Other pathogens may produce extracellular barriers like the biofilm matrix produced by $P$. aeruginosa in the context of chronic lung infections. This biofilm acts as a diffusion barrier, preventing inhaled immunopharmaceutics from reaching their molecular target. Antibody-based fragments, such as fragment antigen-binding (Fab) and single-chain variable fragments $(\mathrm{scFv})$ might be more efficient in crossing over the biofilm, like they penetrate better solid tumors (13), and eradicate $P$. aeruginosa. Secondly, the host physical defenses, which prevent foreign particles from penetrating into the respiratory tract, may limit the accessibility of inhaled immunopharmaceutics to their target. Among them, the mucus and the mucociliary escalator are highly efficient clearance mechanisms $(14,15)$. The development of mucoadhesive formulations may be helpful to enhance the bioavailability of inhaled drugs (16). In contrast, antiadhesive molecules, such as polyethylene glycol may facilitate 
TABLE 1 | Marketed immunotherapeutics and immunoprophylactics for infectious diseases.

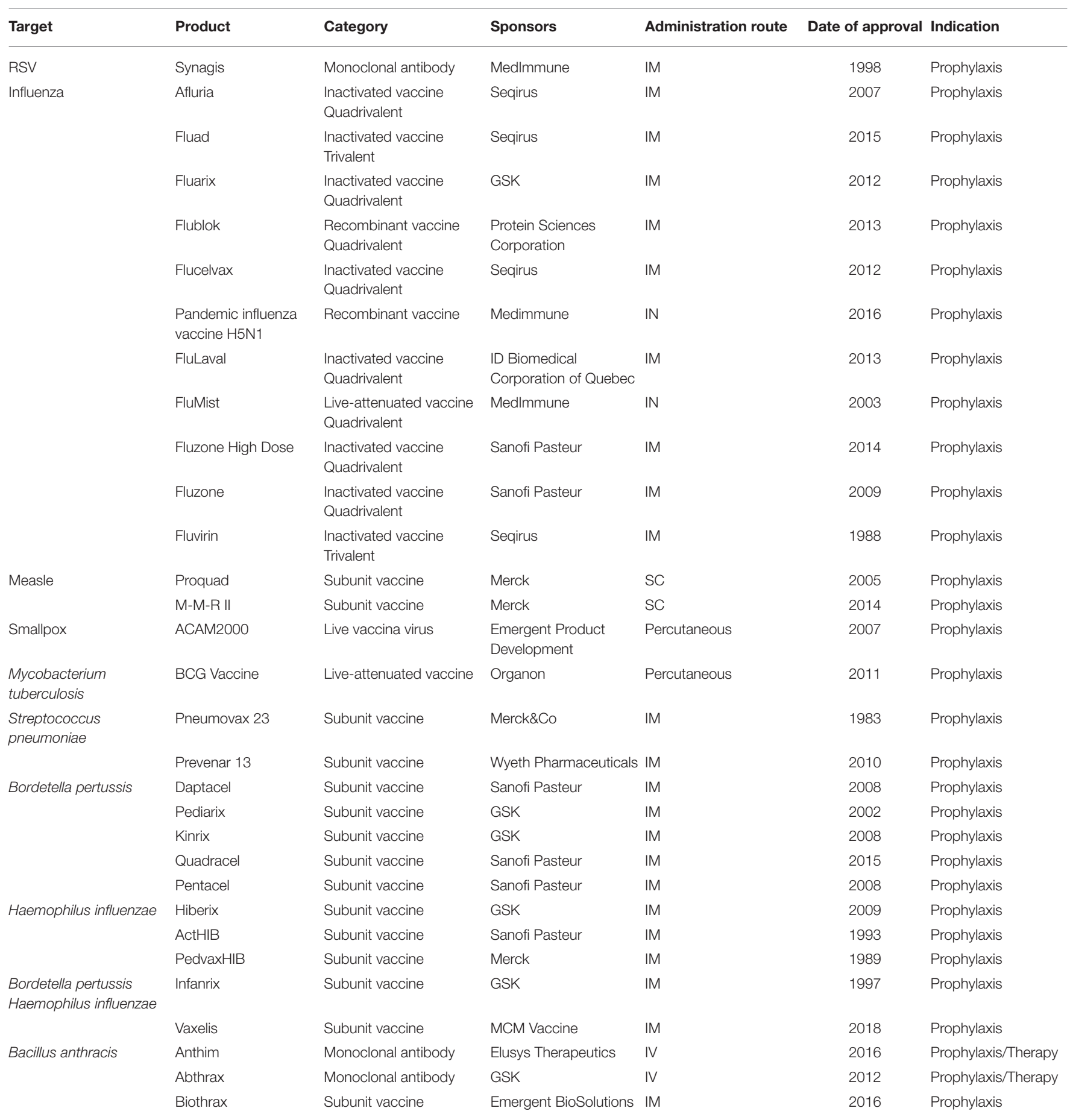

IM, intramuscular; IN, inhalation (nasal); SC, subcutaneous.

immunopharmaceutics translocation through the mucus blanket, as shown in vitro (17) and in vivo (18) for other applications. It is noteworthy that, in some pathological conditions (e.g., chronic sinusitis, CF and COPD), the mucus gets thicker. In $\mathrm{CF}$, the mucus exhibited an increased density of disulfide cross-links, further tightening the mucus mesh space, thereby reinforcing its steric barrier potency to immunopharmaceutics (19). To date, overcoming this physical barrier has not been addressed in the design of inhaled immunopharmaceutics. Other biological barriers include alveolar macrophages and the pulmonary surfactant layer in the alveolar region. While the molecular interactions between inhaled particles and 


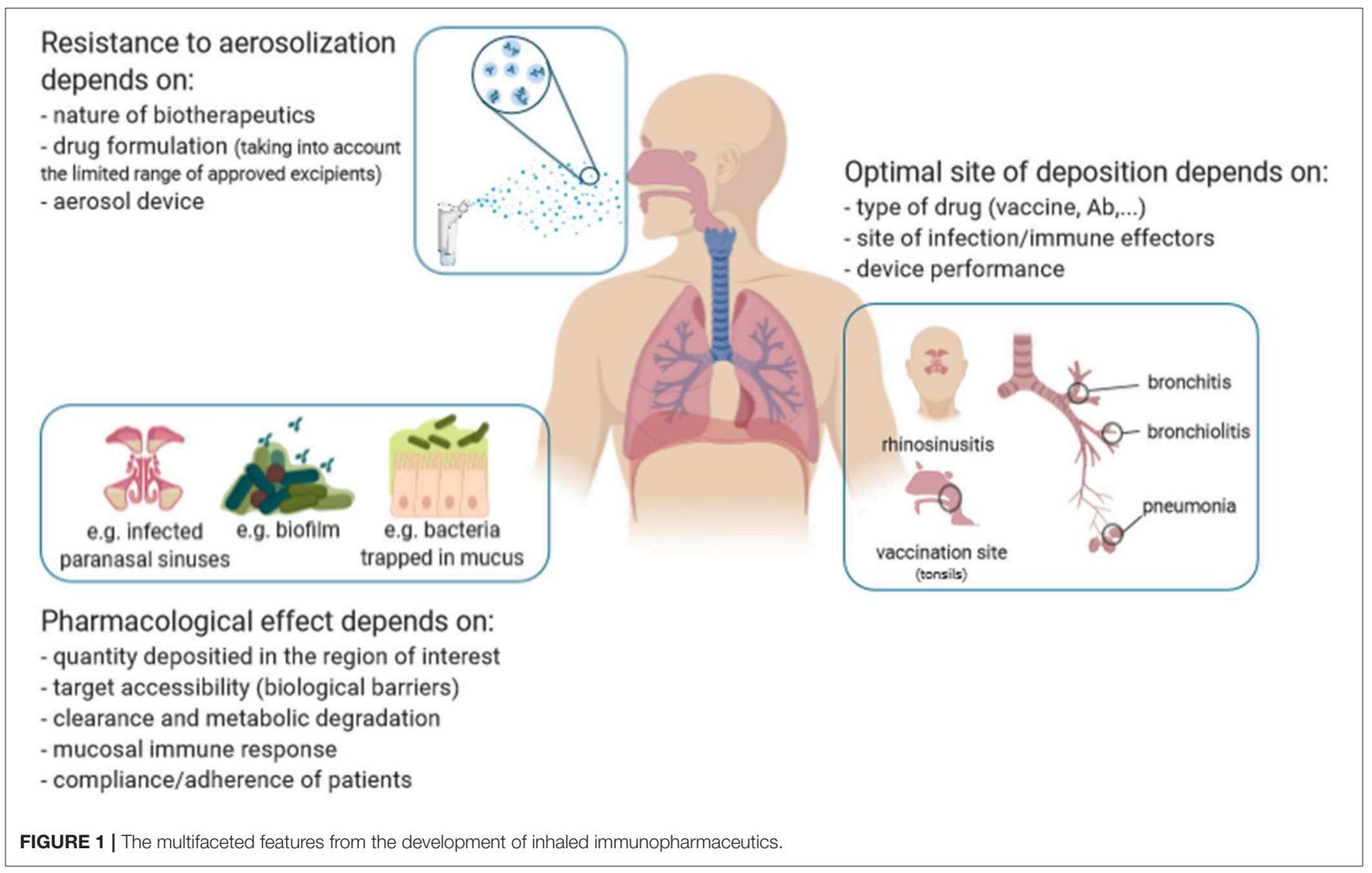

the surfactant are largely unknown, some evidences indicate that surfactant proteins may facilitate the uptake of inhaled particles by alveolar macrophages (20). Alveolar macrophages patrol the airways and phagocytose inhaled organic (including pathogens) and inorganic particles ranging between 0.5 and $5 \mu \mathrm{m}$ (21). Interestingly, the size-discriminating property of their phagocytosis potency has led to the development of innovative approaches for inhaled drugs, in which carrier entrappedparticles of smaller or larger size are inhaled to escape the alveolar macrophage phagocytosis and to provide a better controlled drug release [(22, 23); Figure 1]. This strategy is investigated for mucosal vaccines to prevent the degradation or denaturation of the peptide/antigen, to sustain its release and favor delivery and adjuvancy (24).

The lung mucosa is a metabolic active environment (25). The presence of proteases [which is more prevalent in the nasal mucosa (26)] may degrade therapeutic proteins before they reach their targets. In addition to host enzymes, bacterial pathogens, like $P$. aeruginosa, release additional proteases, which may metabolize respiratory-delivered drugs (27). In this context, the presence of protease inhibitors in the formulation of inhaled protein therapeutics may improve their pharmacokinetics and efficacy, as previously demonstrated for inhaled peptides such as insulin and calcitonin (28). Furthermore, the encapsulation of protein therapeutics into liposomes may also improve stability and reduce the frequency of dosing (29). This strategy has already been clinically validated for the pulmonary delivery of antibiotics (30). Of note, respiratory diseases are often associated with an impairment of the protease/antiprotease balance. In $\mathrm{CF}$, high levels of proteases are a result of the chronic infection and inflammation induced by $P$. aeruginosa (31). This proteolytic environment self-perpetuates the intensity of inflammation, induces mucus hypersecretion and respiratory tissue damage, which may ultimately affect inhaled immunotherapeutics (Figure 1).

\section{CONCLUSION}

Compared to the expansion of biopharmaceutics (excluding non-recombinant vaccines) in all medical areas, the field of inhaled protein therapeutics/vaccines has stagnated, with only few drugs approved so far. Despite promising preclinical data and significant advances on macromolecule inhalation, a definitive demonstration that effective and intact inhaled immunopharmaceuticals could be delivered (topically) to humans is still lacking.

Although, we cannot rule out that the recent failures of inhaled biopharmaceutics (Exubera and ALX-0171) make it challenging, to our opinion, it may be time for thinking carefully where inhalation may have the edge over other routes: "finding the right use for this modality!" They may be many possibilities considering the unmet clinical needs for respiratory 
diseases and the growing market of immunopharmaceutics. But the inhalation route must be envisioned and integrated early taking into account the disease/population, the target, the drug and the device (Figure 1), rather than adapting an approved molecule for the inhalation route. RTIs are undoubtedly an appropriate clinical situation for inhalation, if we consider the importance of matching the delivery of immunoprophylatics or immunotherapeutics to their site of action. Anti-infectious macromolecules may certainly benefit from the success of inhaled antibiotics, but it is critical to remember their precise molecular nature associated with a unique pharmacokinetics profile when considering their development for inhalation. Besides, the recent report of a universal flu vaccine, comprised of Ab-based therapeutics ( $\mathrm{VHH}$ ) produced by an adenoassociated virus delivered intranasally pushed further the boundaries of the potential of the inhalation route for immunoprophylactics (32).

\section{REFERENCES}

1. Dall'Acqua WF, Kiener PA, Wu H. Properties of human IgG1s engineered for enhanced binding to the neonatal Fc receptor (FcRn). J Biol Chem. (2006) 281:23514-24. doi: 10.1074/jbc.M604292200

2. Labiris NR, Dolovich MB. Pulmonary drug delivery. Part I: physiological factors affecting therapeutic effectiveness of aerosolized medications. Br J Clin Pharmacol. (2003) 56:588-99. doi: 10.1046/j.1365-2125.2003.01892.x

3. Larios Mora A, Detalle L, Gallup JM, Van Geelen A, Stohr T, Duprez L, et al. Delivery of ALX-0171 by inhalation greatly reduces respiratory syncytial virus disease in newborn lambs. mAbs. (2018) 10:778-95. doi: 10.1080/19420862.2018.1470727

4. Leyva-Grado VH, Tan GS, Leon PE, Yondola M, Palese P. Direct administration in the respiratory tract improves efficacy of broadly neutralizing anti-influenza virus monoclonal antibodies. Antimicrob Agents Chemother. (2015) 59:4162-72. doi: 10.1128/AAC.00290-15

5. Secher T, Dalonneau E, Ferreira M, Parent C, Azzopardi N, Paintaud $\mathrm{G}$, et al. In a murine model of acute lung infection, airway administration of a therapeutic antibody confers greater protection than parenteral administration. J Control Release. (2019) 303:24-33. doi: 10.1016/j.jconrel.2019.04.005

6. Kang MC, Choi DH, Choi YW, Park SJ, Namkoong H, Park KS, et al. Intranasal introduction of $\mathrm{Fc}$-fused interleukin-7 provides long-lasting prophylaxis against lethal influenza virus infection. J Virol. (2015) 90:2273-84. doi: 10.1128/JVI.02768-15

7. de Swart RL, de Vries RD, Rennick LJ, van Amerongen G, McQuaid S, Verburgh RJ, et al. Needle-free delivery of measles virus vaccine to the lower respiratory tract of non-human primates elicits optimal immunity and protection. NPJ vaccines. (2017) 2:22. doi: 10.1038/s41541-017-0022-8

8. Bodier-Montagutelli E, Mayor A, Vecellio L, Respaud R, Heuze-Vourc'h N. Designing inhaled protein therapeutics for topical lung delivery: what are the next steps? Exp Opin Drug Deliv. (2018) 15:729-36. doi: 10.1080/17425247.2018.1503251

9. Respaud R, Vecellio L, Diot P, Heuze-Vourc'h N. Nebulization as a delivery method for mAbs in respiratory diseases. Exp Opin Drug Deliv. (2015) 12:1027-39. doi: 10.1517/17425247.2015.999039

10. Respaud R, Marchand D, Pelat T, Tchou-Wong KM, Roy CJ, Parent C, et al. Development of a drug delivery system for efficient alveolar delivery of a neutralizing monoclonal antibody to treat pulmonary intoxication to ricin. J Control Release. (2016) 234:21-32. doi: 10.1016/j.jconrel.2016. 05.018

11. Carvalho TC, McConville JT. The function and performance of aqueous aerosol devices for inhalation therapy. J Pharm Pharmacol. (2016) 68:556-78. doi: $10.1111 /$ jphp. 12541

\section{AUTHOR CONTRIBUTIONS}

TS, AM, and NH-V participated in the review of research. $\mathrm{NH}-\mathrm{V}$ prepared figure. TS and AM prepared table. All authors contributed to the manuscript.

\section{FUNDING}

This work was supported by the French National Research Agency as part of the Investissements d'Avenir program (LabEx MAbImprove, ANR-10-LABX-53-01), by the Region Centre Valde-Loire (ARD2020 Biomedicament, PRIMine project/APR IR 2019, Novantinh project), by French Cystic Fibrosis Foundation VLM (Grant No. RF20170502036). TS was funded by a fellowship from ANR-10-LABX-53-01. AM was funded by a CIFRE thesis partnership between the CEPR and Sanofi. Figure was created by BioRender.com.
12. Ho DK, Nichols BLB, Edgar KJ, Murgia X, Loretz B, Lehr CM. Challenges and strategies in drug delivery systems for treatment of pulmonary infections. Eur J Pharm Biopharm. (2019) 144:110-24. doi: 10.1016/j.ejpb.2019.09.002

13. Thurber GM, Schmidt MM, Wittrup KD. Antibody tumor penetration: transport opposed by systemic and antigen-mediated clearance. Adv Drug Deliv Rev. (2008) 60:1421-34. doi: 10.1016/j.addr.2008.04.012

14. Geiser M, Cruz-Orive LM, Im Hof V, Gehr P. Assessment of particle retention and clearance in the intrapulmonary conducting airways of hamster lungs with the fractionator. J Microsc. (1990) 160(Pt 1):75-88.

15. Gizurarson S. The effect of cilia and the mucociliary clearance on successful drug delivery. Biol Pharm Bull. (2015) 38:497-506. doi: 10.1248/bpb.b14-00398

16. Takeuchi H, Yamamoto H, Kawashima Y. Mucoadhesive nanoparticulate systems for peptide drug delivery. Adv Drug Deliv Rev. (2001) 47:3954.doi: $10.1016 / \mathrm{s} 0169-409 \mathrm{x}(00) 00120-4$

17. Lai SK, O'Hanlon DE, Harrold S, Man ST, Wang YY, Cone R, et al. Rapid transport of large polymeric nanoparticles in fresh undiluted human mucus. Proc Natl Acad Sci USA. (2007) 104:1482-7. doi: 10.1073/pnas.0608611104

18. Muralidharan P, Mallory E, Malapit M, Hayes D Jr, Mansour HM. Inhalable PEGylated phospholipid nanocarriers and PEGylated therapeutics for respiratory delivery as aerosolized colloidal dispersions and dry powder inhalers. Pharmaceutics. (2014) 6:333-53. doi: 10.3390/pharmaceutics6020333

19. Yuan S, Hollinger M, Lachowicz-Scroggins ME, Kerr SC, Dunican $\mathrm{EM}$, Daniel BM, et al. Oxidation increases mucin polymer crosslinks to stiffen airway mucus gels. Sci Transl Med. (2015) 7:276ra27. doi: 10.1126/scitranslmed.3010525

20. Chroneos ZC, Sever-Chroneos Z, Shepherd VL. Pulmonary surfactant: an immunological perspective. Cell Physiol Biochem. (2010) 25:13-26. doi: $10.1159 / 000272047$

21. Geiser M. Update on macrophage clearance of inhaled micro- and nanoparticles. J Aerosol Med Pulm Drug Deliv. (2010) 23:207-17. doi: 10.1089/jamp.2009.0797

22. Pudlarz AM, Czechowska E, Ranoszek-Soliwoda K, Tomaszewska E, Celichowski G, Grobelny J, et al. Immobilization of recombinant human catalase on gold and silver nanoparticles. Appl Biochem Biotechnol. (2018) 185:717-35. doi: 10.1007/s12010-017-2682-2

23. Tsapis N, Bennett D, Jackson B, Weitz DA, Edwards DA. Trojan particles: large porous carriers of nanoparticles for drug delivery. Proc Natl Acad Sci USA. (2002) 99:12001-5. doi: 10.1073/pnas.182233999

24. Osman N, Kaneko K, Carini V, Saleem I. Carriers for the targeted delivery of aerosolized macromolecules for pulmonary pathologies. Exp Opin Drug Deliv. (2018) 15:821-34. doi: 10.1080/17425247.2018.1502267

25. Candiano G, Bruschi M, Pedemonte N, Musante L, Ravazzolo R, Liberatori $\mathrm{S}$, et al. Proteomic analysis of the airway surface liquid: modulation by 
proinflammatory cytokines. Am J Physiol Lung Cell Mol Physiol. (2007) 292:L185-98. doi: 10.1152/ajplung.00085.2006

26. Zhou XH. Overcoming enzymatic and absorption barriers to non-parenterally administered protein and peptide drugs. J Control Release. (1994) 29:239-52. doi: 10.1016/0168-3659(94)90071-X

27. Gellatly SL, Hancock RE. Pseudomonas aeruginosa: new insights into pathogenesis and host defenses. Pathogens Dis. (2013) 67:159-73. doi: 10.1111/2049-632X.12033

28. Hussain A, Arnold JJ, Khan MA, Ahsan F. Absorption enhancers in pulmonary protein delivery. J Control Release. (2004) 94:15-24. doi: 10.1016/j.jconrel.2003.10.001

29. Gibbons AM, McElvaney NG, Taggart CC, Cryan SA. Delivery of rSLPI in a liposomal carrier for inhalation provides protection against cathepsin L degradation. J Microencapsul. (2009) 26:513-22. doi: 10.1080/02652040802466535

30. Haworth CS, Bilton D, Chalmers JD, Davis AM, Froehlich J, Gonda I, et al. Inhaled liposomal ciprofloxacin in patients with non-cystic fibrosis bronchiectasis and chronic lung infection with Pseudomonas aeruginosa (ORBIT-3 and ORBIT-4): two phase 3, randomised controlled trials. Lancet Respir Med. (2019) 7:213-26. doi: 10.1016/S2213-2600(18)30427-2
31. Twigg MS, Brockbank S, Lowry P, FitzGerald SP, Taggart C, Weldon $\mathrm{S}$. The role of serine proteases and antiproteases in the cystic fibrosis lung. Mediators Inflamm. (2015) 2015:293053. doi: 10.1155/2015/2 93053

32. Laursen NS, Friesen RHE, Zhu X, Jongeneelen M, Blokland S, Vermond J, et al. Universal protection against influenza infection by a multidomain antibody to influenza hemagglutinin. Science. (2018) 362:598-602. doi: 10.1126/science.aaq0620

Conflict of Interest: The authors declare that the research was conducted in the absence of any commercial or financial relationships that could be construed as a potential conflict of interest.

Copyright (C) 2019 Sécher, Mayor and Heuzé-Vourc'h. This is an open-access article distributed under the terms of the Creative Commons Attribution License (CC BY). The use, distribution or reproduction in other forums is permitted, provided the original author(s) and the copyright owner(s) are credited and that the original publication in this journal is cited, in accordance with accepted academic practice. No use, distribution or reproduction is permitted which does not comply with these terms. 